\title{
Effect of Diabetes Mellitus on the Respiratory Muscle Power in Sudanese Diabetic Patients
}

\author{
Elmutaz H Taha ${ }^{1}$, Ibrahim A Ali ${ }^{*}$ and Omer A Musa ${ }^{2}$ \\ ${ }^{1}$ Department of Physiology, Faculty of Medicine, Dongola University, Sudan. \\ ${ }^{2}$ Department of Physiology, Faculty of Medicine, National Ribat University, Sudan
}

\begin{abstract}
Received: August 21, 2018; Accepted: September 14, 2018; Published: September 19, 2018
*Corresponding author: Ibrahim A Ali, Department of Physiology, Faculty of Medicine, The National Ribat University, Khartoum, Sudan. Email: hemamedicine@gmail.com
\end{abstract}

\begin{abstract}
Background: Diabetes mellitus is a leading cause of illness and death. Respiratory muscle endurance is of interest in pulmonary, critical care and many other areas of medicine. Reduced muscle strength has been reported in diabetic patients.

Methods: A cross sectional study was conducted in The National Ribat Teaching Hospital and Jabir Abualiz Specialized Diabetes Center in Khartoum state during 2008-2009 to measure the respiratory muscle power in 31 type II diabetic patients (case group) and 30 nondiabetics patients (control groups). Respiratory muscle power was measured by using Respiratory Pressure Meter device (MicroRPM).
\end{abstract}

Results: Diabetic patients showed significant reduction in Maximal Expiratory Pressure (MEP) $71.8 \pm 7$ compared to control 101.03 \pm 4.7 . Depending on exercise performance diabetic patients with exercise performance revealed a significant increase in MEP compared with those without exercise. There were no significant differences in MEP among diabetics patients according to vitamin supplementary; Although there is slight increase in those with vitamin supplementary.

Conclusion: This study has shown that type II DM decreases the respiratory muscle power which could be predisposing to more respiratory problems. Exercise and well control of diabetes helped in preserving normal respiratory muscle power.

Key Words: Diabetes mellitus; Respiratory Muscle Power; Sudan; Reduced Muscle Strength; Diabetic Patients;

\section{Introduction}

Diabetes mellitus is a leading cause of illness and death. Respiratory muscle endurance is of interest in pulmonary, critical care and many other areas of medicine. Reduced muscle strength has been reported in diabetic patients. Bilateral or unilateral diaphragmatic paralysis has been observed in diabetic patients $[1,2]$. In addition, Meo et al conducted a study and determined the respiratory muscles endurance by a direct MVV test during inspiratory and expiratory phases of respiration by using a MP100 student Bio Pac system [3]. They reported that the respiratory muscles endurance was impaired, and a greater perception of respiratory exertion was noticed in diabetic patients relative to their matched controls. Moreover, breathlessness on exertion and orthopnea in association with Type 2 diabetes mellitus has been also reported [4]. Diabetes mellitus is also associated with poor skeletal muscle strength due to increased protein catabolism [5]. For this reason respiratory muscle endurance also decreases in diabetes mellitus [6].

The increased ratio of FEV1/ FVC (\%) in diabetic patients of longer duration is due to disproportionate reduction of FVC and FEV1, which indicate that long-standing hyperglycemia may cause predominantly restrictive type of lung disorder. All these changes may be due to glycation of the chest wall and bronchial tree protein. This is further supported by negative correlation of FVC and FEV1 and positive correlation of FEV1/FVC\% with longer duration of diabetes. The negative correlation of FVC and FEV1 with duration of diabetes indicate that long standing hyperglycemia may intensify the devastating effect of the disease [6].

The present study was designed to observe the effect of diabetes mellitus on lung function and respiratory muscle power in patients with diabetes mellitus belonging to specific society namely Sudanese in the center of Sudan.

\section{Materials and Methods}

A cross sectional study was conducted to measure the respiratory muscle power in 31 diabetic patients (case group) and 30 non-diabetics patients (control groups) in Khartoum state in Sudan. The study was conducted in The National Ribat Teaching Hospital and Jabir Abualiz Specialized Diabetes Center which are located in Khartoum state- the capital of Sudan. This study group includes all Sudanese diabetic patients attending to the diabetic clinics in The National Ribat Teaching Hospital and Jabir Abualiz Specialized Diabetes Center during the period of study. Age range from 20 to 75 years, and mean duration of disease was $10.60 \pm$ 1.11 years. Their duration of disease ranged from 1 to 30 years. Patients with diabetes were individually matched with controls for age, height and weight. Matching between both groups was within \pm 3 years for age, \pm 4 centimeters for height and \pm 5 kilograms for weight. Overall, there were no significant differences in anthropometric means in combined or stratified data. Age and height were given more emphasis for matching, as these 
two relate better to lung function than weight. 11 Controls were selected from a similar community with the same socioeconomic status relative to patients with diabetes. All subjects had never smoked. All subjects completed a questionnaire, which included anthropometric data and a consent form. Subjects with gross abnormalities of the vertebral column or thoracic cage, known history of acute or chronic respiratory infections, neuromuscular disease, malignancy, cardiopulmonary disease or a history of major abdominal or chest surgery were excluded from the study. In addition, subjects with current or previous drug or tobacco (smoked or chewed) addictions were excluded. Patients with complications of diabetes such as neuropathy, nephropathy and retinopathy were also excluded from the study.

\section{Technique of Maximal Expiratory Pressure (MEP)}

Respiratory muscle power was measured by using Respiratory Pressure Meter device (MicroRPM).The MicroRPM brings together the measurements of Maximum Inspiratory and Expiratory Mouth Pressures (MIP/MEP) with Sniff Nasal Inspiratory Pressure (SNIP) in one instrument. The MicroRPM is easy to use, operated by a simple three setting slide bar (Off, MIP/ MEP, SNIP) and comes in a sturdy carry case. It is battery operated with a clear digital display of the results in $\mathrm{CmH2O}$, using the latest piezo resistive pressure sensing technology for accurate and reliable measurement; see figure (1). Maximal Expiratory Pressure at the mouth is measured at TLC (Total Lung Capacity), lungs full. The patient was asked to blow through the mouth piece starting from maximum lung capacity. Patient inhales air as maximum as possible, then forcibly blows through the mouth piece for at least 5 seconds. The maximum expiratory mouth pressure will be displayed digitally in $\mathrm{cm} / \mathrm{H} 2 \mathrm{O}$. After explaining the steps of the test to the patients, the maneuver was repeated three times with a suitable 1 or 2 minute recovery period between efforts and the best reading was taken as the value of expiratory muscle strength.

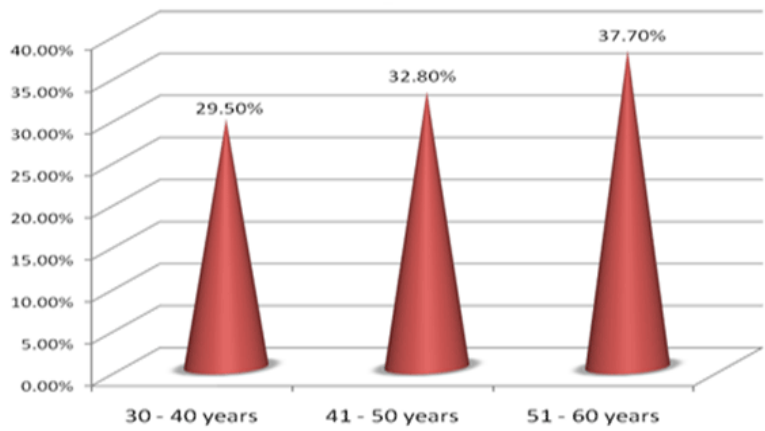

Figure 1: Age distribution among study population.

\section{Data analysis}

Data was analyzed by Statistical Package for Social Science (SPSS) version 20 and any significance different between case and control groups in respiratory muscle power were identified by using t-test among sociodemographic and medical characteristic. Data had been displayed by tables and histogram.

\section{Ethical clearance}

Ethical clearance was obtained from the scientific committee of the university and from the Hospital and informed consent was taken from the patients.

\section{Results}

Statistical test to confirm the data normality was carried out and the data was found to be normally distributed by kolmogorovsmirnov test.

In this study 61 adult subjects were included, 54.10\% (34) male and $45.9 \%$ (27) female. 31 diabetic (17 males and 14 females) and 30 control (17 male and 13 female).

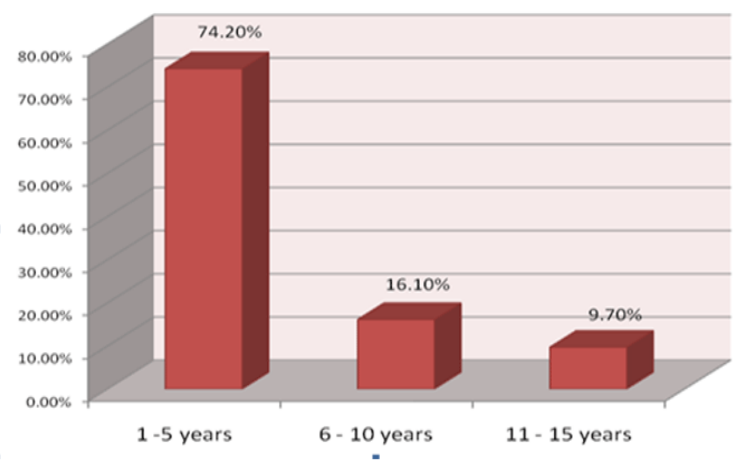

Figure 2: Duration of diabetes mellitus among case group.

Figure (2) showed that most of diabetic patients $74.2 \%$ (23 patients) included in this study have DM for $1-5$ years. Patients with DM for more than 10 years (3 patients) have been well controlled on regular treatment and perform exercise daily for at least one-hour walking for the last 10 years compared to the rest.

Figure (2) showed that most of the subjects $37.7 \%$ (23 subjects) were in the age group 51- 60 years, $32.8 \%$ (20 subjects) were in the age group $41-50$ years and $29.5 \%$ (18 subjects) were in the age group 30-40 years.

Table (1) Summarizes the comparison of the respiratory muscle power between diabetic patients and their matched control group.

Table 1: Maximum expiratory pressure (MEP) and anthropometric data of total diabetic patients (31) compared with their matched controls (30).

\begin{tabular}{|c|c|c|c|c|}
\hline Parameters & $\begin{array}{c}\text { DM } \\
\text { mean +- SEM } \\
\mathbf{n} \text { (31) }\end{array}$ & $\begin{array}{c}\text { Control } \\
\text { Mean +- SEM }\end{array}$ & P. value & \\
\hline Age (years) & $36.1+-7.47$ & $36.8+-6.81$ & .823 & Ns \\
\hline Height (cm & $168.73+-2.142$ & $168+-9.243$ & .930 & Ns \\
\hline Weight (kg & $70.7+-3$ & $78.233+-2.25$ & .121 & Ns \\
\hline MEP(cm $\backslash$ H2o) & $90.92+-6.536$ & $101.033+-4.7$ & .19 & Ns \\
\hline
\end{tabular}


Table (2) Summarizes the comparison of the maximum expiratory pressure between diabetic patients with duration of disease 1-10 years and their matched control group. However, diabetic patients showed significant reduction in PEFR and MEP.

Table 2: MEP and anthropometric data for diabetic patients with duration of disease 1-10 years compared with their matched controls.

\begin{tabular}{|c|c|c|c|c|}
\hline \multirow{2}{*}{ Parameters } & $\begin{array}{c}\text { DM } \\
\text { mean +- SEM } \\
\mathbf{n ~ ( 2 8 )}\end{array}$ & $\begin{array}{c}\text { Control } \\
\text { Mean +- SEM } \\
\mathbf{n ~ ( 3 0 )}\end{array}$ & P. value & \\
\cline { 1 - 3 } & $35.75+-7.47$ & $36.8+-6.81$ & .786 & Ns \\
\hline Height (cm & $164.65+-2.142$ & $168+-9.243$ & .430 & Ns \\
\hline Weight (kg & $69.4+-3$ & $78.233+-2.25$ & .101 & Ns \\
\hline MEP(cm\H2o) & $75.55+-6.536$ & $101.033+-4.7$ & .008 & S \\
\hline & & & & \\
\hline
\end{tabular}

Table (3) Summarizes the comparison of the Maximum expiratory pressure (MEP) between diabetic patients with duration of disease 1- 5 years and their matched control group. However, diabetic patients showed a significant reduction in PEFR and MEP.

Diabetic patients showed significant reduction in MEP 71.8 +-7 compared to control $101.03+-4.7$. There was no significant difference in pulmonary function and anthropometric data .

\begin{tabular}{|c|c|c|c|c|}
\hline Parameters & $\begin{array}{c}\text { DM } \\
\text { mean +- SEM } \\
\text { n (23) }\end{array}$ & $\begin{array}{c}\text { Control } \\
\text { Mean +- SEM } \\
\text { n (30) }\end{array}$ & P. value & \\
\hline Age (years) & $35.1+-7.47$ & $36.8+-6.81$ & .723 & Ns \\
\hline Height (cm & $166.7+-2.142$ & $168+-9.243$ & .630 & Ns \\
\hline Weight (kg & $74+-3$ & $78.233+-2.25$ & .221 & Ns \\
\hline $\operatorname{MEP}(\mathrm{cm} \backslash \mathrm{H} 2 \mathrm{o})$ & $79.30+-6.536$ & $101.033+-4.7$ & .008 & S \\
\hline
\end{tabular}

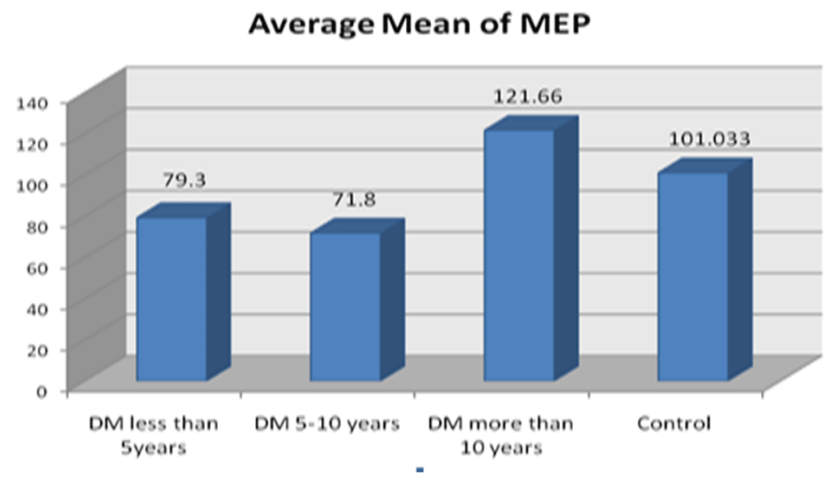

Figure 3: The average mean of MEP among diabetic patients with different duration of disease and control group.
Exercise activity in case group (diabetic) was low with percentage $45.2 \%$ (14 patients), while $54.8 \%$ (17 patients) are not performing exercise (figure 4 ).

Patients, who perform exercise, walk for half an hour, but not regularly. However, three patients with duration of disease more than 10 years perform exercise (walking) for at least one hour daily for more than 10 years. (Table 4 )

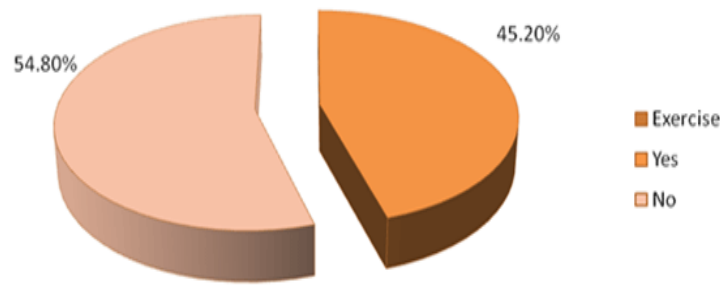

Figure 4: Exercise performance among diabetic patients

Table 4: MEP among diabetic patients with exercise performance and those without performance.

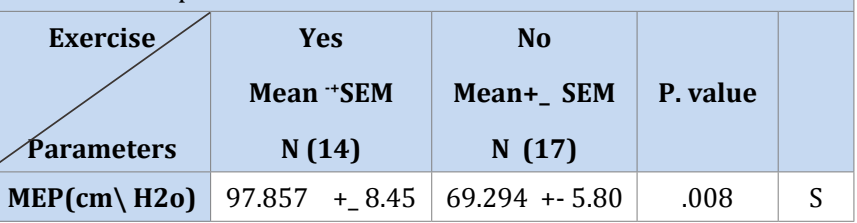

Depending on exercise performance diabetic patients with exercise performance revealed a significant increase in MEP compared with those without exercise.

There was no significant difference among the means of MEP between the diabetic patients with exercise performance compared with their matched control as shown in table (5).

Table 5: MEP among diabetic patients with exercise performance compared with their matched control.

\begin{tabular}{|l|c|c|c|c|}
\hline Exercise & Yes & Control & \\
\hline Parameters & $\mathrm{N}(14)$ & $\mathrm{n}(30)$ & & \\
\hline $\mathrm{MEP}(\mathrm{cm} \backslash \mathrm{H} 2 \mathrm{o})$ & $97.857+{ }^{+} \mathrm{SEM}$ & Mean +- SEM & P. value & \\
\hline
\end{tabular}

Depending on exercise performance diabetic patients without exercise performance revealed a significant reduction in MEP and PEFR compared with their matched control as shown in table (6).

Table 6: MEP among diabetic patients without exercise performance compared with their matched control.

\begin{tabular}{|l|c|c|c|c|}
\hline Exercise & No & Control & \\
& Mean+_SEM & Mean +- SEM & P. value & \\
\hline Parameters & $\mathrm{N}(17)$ & $\mathrm{n}(30)$ & & \\
\hline $\mathbf{M E P}(\mathbf{c m} \backslash \mathbf{H 2 0})$ & $69.294+-5.80$ & $101.03+-4.7$ & .009 & $\mathrm{~S}$ \\
\hline
\end{tabular}


According to vitamin supplementary most of the diabetic patients $61.30 \%$ (19 patients) don't use vitamin, while $38.70 \%$ (12 patients) use vitamin regularly as shown in figure (5).

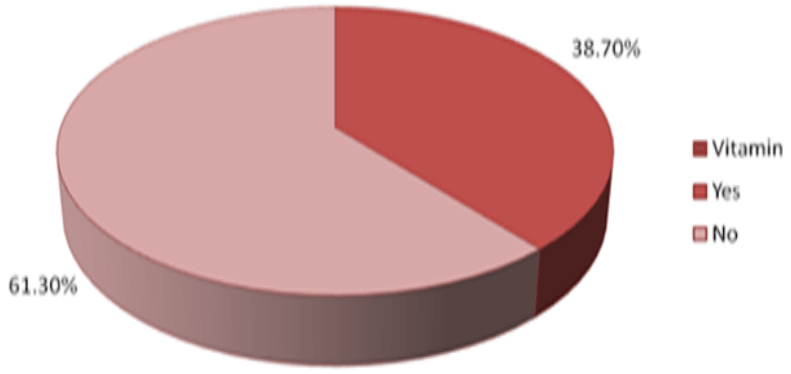

Figure 5: Vitamin supplementary status among diabetic patients.

Table (7) illustrated there were no significant differences in MEP among diabetics patients according to vitamin supplementary .Although there is slight increase in those with vitamin supplementary.

\begin{tabular}{|c|c|c|c|}
\hline Parameters & $\begin{array}{c}\text { Yes } \\
\text { Mean }^{++} \text {SEM } \\
\text { n (12) }\end{array}$ & $\begin{array}{c}\text { No } \\
\text { Mean }{ }^{+-} \text {SEM } \\
\text { n (19) }\end{array}$ & P. value \\
\hline$M E P(\mathrm{~cm} \backslash \mathrm{H} 20)$ & $83.5^{+-} 7.74$ & $81.368^{+-} 7.74$ & .855 \\
\hline
\end{tabular}

\section{Discussion}

This study was conducted among type II diabetic patients (31) and their control (30). The main findings from this study reveal that the respiratory muscle power of the diabetic patients was markedly reduced in relation to their control.

Diabetic patients with duration of disease from 5 to 10 years revealed a significant reduction ( $p$ value $<.05$ ) in their MEP (71.8 +-7) compared to their control group (101.3+- 4.7). In this study the respiratory muscle power of diabetic patients tends to decrease with the increased duration of the disease. This is in accordance with findings of Park Sw et al who reported that diabetes mellitus is associated with poor skeletal muscle strength due to increased protein catabolism [5]. According to exercise diabetic subjects without exercise performance showed significant reduction in respiratory muscle power (69.3+- 5.8) compared to diabetic patients with exercise performance (97.9+8.4). On the other hand there was slight increase (not significant) in pulmonary function data. This is in agreement with the findings of Leem Romer et al who reported that in athlete' s racing bicycle, respiratory muscle power was increased by $35 \mathrm{~W}$ every $3 \mathrm{~min}$ starting from $95 \mathrm{~W}$ [7]. No significant values were obtained in diabetic patients with vitamin supplementary on the pulmonary function tests and respiratory muscle power compared with diabetic patients without vitamin supplementary. As the number is limited, more diabetic should be included in another study.

\section{Conclusion}

This study has shown that type II DM decreases the respiratory muscle power which could be predisposing to more respiratory problems. Exercise and well control of diabetes helped in preserving normal respiratory muscle power. This could be considered a pilot study and more investigation of more numbers of DM with different duration of disease and level of exercise needs to be performed. A short study to needed to follow the outcome of DM and RMP in relation to pulmonary problems. The study should be repeated by measuring inspiratory muscle power (MIP), as MEP reflexes the expiratory muscle power only. Continuous reasonable exercise with good control is highly recommended for all diabetics.

\section{References}

1. Andersen $\mathrm{H}$, Poulsen $\mathrm{PL}$, Mogensen E and Jakobsen J. Isokinetic muscle strength in long-term IDDM patients in relation to diabetic complications. Diabetes. 1996;45(4):440-445.

2. Kadosh S, Qupti G and Flatau E. Unilateral diaphragmatic paralysis in a diabetes patient; Harefuah. 2005;144(12):834-835, 911.

3. Meo S, Abdul Majeed AlDrees, Muhammad Arif and Khalid Al-Rubean. Assessment of respiratory muscles endurance in diabetic patients. Saudi Medical Journal. 2006;27(2):223-226.

4. White E, Bullock E, Hudgson P, Home D and Gibson J. Phrenic neuropathy in association with diabetes. Diabet Med. 1992;9(10):954956.

5. Park SW, Goodpaster BH, Strotmeyer ES, Rekeneire N, Harris TB and Schwartz AN. et al. Decreased Muscle Strength and Quality in Older Adults With Type 2 Diabetes. The Health, Aging and Body Composition Study. Diabetes. 2006;55(6):1813-1818.

6. Sultan A. Meo, Abdul Majeed Al Drees, Jehangeer Ahmed, Sayed Fayaz Ahmed Shah, Khalid Al-Regaiey and Ashraf Husain. et al. Effect of Duration of Disease on Ventilatory Function in an Ethnic Saudi Group of Diabetic Patients. J Diabetes Sci Technol. 2007;1(5):711717. Doi: 10.1177/193229680700100515.

7. Tobin MJ, Chadha TS, Jenouri G, Birch SJ, Gazeroglu HB and SacknerMA. Breathing patterns. Diseased subjects. Chest. 1983;84(3):286-294. 